\title{
Association between estimated glomerular filtration rate and outcomes in patients with diabetic foot ulcers: a 3-year follow-up study
}

\section{Yang He', Hongjie Qian'1, Lei Xu1', Shanshan Zhang'1, Xueming Gu², Junyi Gu², Jianyuan Shi ${ }^{2}$, Yaping Shen ${ }^{2}$, Jianmin Liu' and Zhengyi Tang ${ }^{1,2}$}

${ }^{1}$ Shanghai Clinical Center for Endocrine and Metabolic Diseases, Yuanyang Subdivision for Diabetic Foot Disease, Shanghai Institute of Endocrine and Metabolic Diseases, Ruijin Hospital Affiliated to Shanghai Jiaotong University School of Medicine, Shanghai, China and 'Department of Internal Medicine, Shanghai Yuanyang Hospital, Shanghai, China
Correspondence should be addressed to Z Tang

Email

tsy2zyp@163.com

\begin{abstract}
Objective: End-stage renal disease and dialysis have been proven to be associated with poor prognoses in diabetic foot ulcers (DFUs). However, it has rarely been reported whether and to what extent milder renal insufficiency affects the prognosis. The purpose of this study was to investigate the categorized impact of estimated glomerular filtration (eGFR) on the outcomes of patients with DFU.

Design and methods: Three hundred and sixty-six DFU patients hospitalized in a Chinese tertiary hospital were recruited and classified into 4 groups according to the eGFRs as follows: normal ( $\geq 90)$, mildly reduced (60-89), moderately reduced (30-59), and severely reduced $(<30)$. These patients were followed-up for an average of 37 months to observe the outcomes, including ulcer healing, amputation, ulcer recurrence, cardiac or cerebrovascular events and death. The associations between eGFR and the outcomes were analysed by Cox proportional-hazards models.

Results: Compared to patients with normal eGFR, patients with moderately reduced eGFR had higher risk of healing failure (hazard ratio $(\mathrm{HR})=2.08,95 \%$ confidence interval $(\mathrm{Cl}): 1.13-3.82)$, cardiac events $(\mathrm{HR}=5.25,95 \% \mathrm{Cl}$ : $2.17-$ $12.89)$ and death ( $\mathrm{HR}=3.54,95 \% \mathrm{Cl}: 1.36-9.20)$. Severely reduced eGFR was associated with higher incidence of healing failure ( $\mathrm{HR}=2.84,95 \% \mathrm{Cl}: 1.25-6.49)$ and death ( $\mathrm{HR}=4.45,95 \% \mathrm{Cl}: 1.23-16.07)$. The impact of eGFR on ulcer recurrence and cerebrovascular events was not observed in all groups.

Conclusions: Moderately and severely reduced eGFR in patients with DFU were independent predictors for poor prognoses of both the limbs and the patients.
\end{abstract}

\section{Introduction}

Diabetic foot ulcer (DFU), which is a highly prevalent condition in China and worldwide (1), is an advanced complication of diabetes with unfavorable outcomes $(2,3)$.

Diabetes is known to be a leading cause of chronic kidney disease, resulting in renal insufficiency of varying degrees complicated with DFU (4). End-stage renal disease or dialysis was previously suggested to be associated
() 2017 European Society of Endocrinology Printed in Great Britain with poor prognoses of $\operatorname{DFU}(5,6)$. However, the impact of milder renal insufficiency on DFU outcomes has rarely been reported. Although Ghanassia et al. (3) and Morbach et al. (7) noticed the adverse effects of chronic renal insufficiency in the long-term prognosis of DFU patients, renal insufficiency in these reports was defined based on serum creatinine or creatinine clearance (calculated from the Cockcroft-Gault formula), both of 
which are less accurate $(8,9)$ in assessing renal function compared with eGFR calculated using the abbreviated Modification of Diet in Renal Disease (MDRD) formula. Furthermore, none of these studies made a stratification analysis and categorized the impact of each stage of renal insufficiency.

The aim of this study was to investigate the association between renal insufficiency, which was defined and classified based on eGFR, and the outcomes in patients with DFU.

\section{Subjects and methods}

\section{Patient population and selection}

From April 2009 to March 2012, patients hospitalized in our department for DFU were consecutively recruited into the cohort. Those patients with a previous occurrence of DFUs or major amputations were excluded. Patients were followed-up every 6 months from enrolment until March 2014, or until death. The outcomes information was obtained by conducting questionnaire surveys on patients in our department for inpatient or outpatient foot care or by calling their family members over the phone if patients could not be reached because they were receiving foot care elsewhere. All the foot care and outcome assessments in our department were performed by the same treatment team according to the guidelines for diabetic foot treatments recommended by the International Working Group on the Diabetic Foot (IWGDF) $(10,11,12)$.

All patients gave consent for participation in this study. This study protocol was approved by the ethics committee of the Ruijin Hospital affiliated to the Shanghai Jiao Tong University of Medicine.

\section{Definition and measurement of exposure, outcomes and impact factors}

Renal function was assessed based on eGFR. Blood samples for serum creatinine test were taken from venous blood after patients were fasted for at least 8 hours. EGFR was calculated using the following MDRD equation for Chinese adults (13): eGFR $=175 \times$ (serum creatinine $(\mathrm{mg} / \mathrm{dL}))^{-1.234} \times \mathrm{age}^{-0.179} \times 0.79$ (if female). Patients were categorized into 4 groups as follows: normal eGFR: $\geq 90 \mathrm{~mL} / \mathrm{min}$ per $1.73 \mathrm{~m}^{2}$, mildly reduced eGFR: $60-89 \mathrm{~mL} /$ min per $1.73 \mathrm{~m}^{2}$, moderately reduced eGFR: $30-59 \mathrm{~mL} /$ min per $1.73 \mathrm{~m}^{2}$, and severely reduced eGFR: $<30 \mathrm{~mL} / \mathrm{min}$ per $1.73 \mathrm{~m}^{2}$.
The ulcer-related outcomes included primary ulcer healing, ulcer recurrence and amputations, whereas the patient-related outcomes included cardiac events, cerebrovascular events and death. Primary ulcer healing was determined by the full coverage of skin or crusts on the primary wound without major amputation of the limb. Ulcer recurrence was defined as the reappearance of the ulcer after primary wound healing was achieved. An amputation was considered major when it was performed above the tarsometatarsal articulation level (14); otherwise, it was viewed as a minor one. The cardiac events included acute heart failure, acute coronary syndrome and sudden cardiac death. The cerebrovascular events included fatal and non-fatal stroke. The outcome occurrences were recorded in the medical files and death records in our department, or they were obtained through telephonic interviews with patients or their relatives using questionnaires.

Other impact factors, including demographic data, BMI, type and duration of diabetes, HbA1c, severity of DFUs and microvascular or macrovascular comorbidities of the patients were recorded and assessed on admission. HbA1c was measured using high-performance liquid chromatography. DFUs were assessed using the Wagner classification system (15). Wounds at Wagner Grade 1 or 2 were further categorized as mild foot ulcers, whereas the remaining (Wagner 3, 4 and 5) ulcers were regarded as critical ulcers. Diabetic peripheral neuropathy (DPN) was diagnosed when 2 of the following criteria were met (3, 16): (1) neuropathic pain, anesthesia or other symptoms of paresthesia; (2) abnormal pinprick sensation of the lower limbs or altered 10-g Semmes-Weinstein monofilament test; or (3) diminished ankle reflexes. Peripheral artery disease (PAD) was diagnosed if one or more lower limb artery occlusions were spotted by Doppler ultrasound and/or ankle-brachial pressure index $(\mathrm{ABI})<0.9$ in either of the limbs (7). History of coronary heart disease was confirmed by medical records or defined by the presence of a history of angina pectoris or myocardial infarction, any positive cardiac stress test result, or pathological signs on coronary angiography (17). History of stroke was defined as the presence of any neurologic deficiency event with or without sequelae (17). Hypertension was determined by $\mathrm{BP} \geq 140 / 90 \mathrm{mmHg}$ or the current use of antihypertensive medicine.

\section{Statistical analysis}

Quantitative variables were described by the mean \pm S.D. or median (range) according to their distribution, 
and one-way ANOVA was used to make comparisons among groups. Discontinuous variables were expressed using frequency, and comparisons were made using the $\chi^{2}$ test. To analyze the association between reduced eGFR categories and outcomes, hazard ratios with 95\% confidence intervals were first calculated using univariate Cox proportional-hazards models. Then, the following factors were included as confounders to ascertain hazard ratios for ulcer-related outcomes in multiple Cox regression models: age, sex, Wagner grade, PAD, DPN, HbA1c and treatment at baseline. Regarding patientrelated outcomes, disease history, including duration of diabetes, history of coronary heart disease, history of stroke and cardiac treatment at baseline were further added to the multiple Cox proportional hazards models. The accumulated survival rates in the different groups were graphed by the Kaplan-Meier curve, and the logrank test was performed to make comparisons among groups. All the statistical analyses were performed using the SAS statistical system (version 8.0; SAS Institute Inc., Cary, NC). A $P$ value $<0.05$ was considered statistically significant.

\section{Results}

\section{Population assessment and validation}

A total of 366 hospitalized patients with DFU were enrolled in this study. The median age was 69 (range 31-96) years, $62 \%$ were male and $99.2 \%$ had type 2 diabetes. The baseline characteristics of the categorized eGFR groups are given in Table 1. At the initiation of the study, patients with lower eGFR tended to be older, had lower HbA1c values and were more likely to have DPN, history of coronary heart disease, stroke and hypertension. Other characteristics at baseline were similar among study groups.

The treatment information is listed in Table 2. More than $90 \%$ of patients received insulin therapy to control blood glucose. Antiplatelet agents were taken by approximately $50 \%$ of patients. An increasing trend in the use of diuretics and angiotensin converting enzyme inhibitors (ACEI)/angiotensin receptor blocker (ARB) agents was seen with the decrease of eGFR. Only 14 patients received revascularization therapy in other hospitals prior to this study. No revascularization therapy was performed in this study.

The mean length of follow-up was 37 months (range 0.1-60 months). Follow-up data were obtained for $333(91.0 \%)$ participants. The distribution of patients among eGFR categories was not significantly different in the total participant population and in those who had been followed-up (total, 60.7, 25.1, 9.3 and 4.9\%; followed-up, 60.4, 24.9, 9.3 and 5.4\%, respectively; $P=0.99$ ).

\section{Primary ulcer healing}

Of the 317 participants without major amputation and with available follow-up data, 259 (81.7\%) attained primary ulcer healing by the end of the study.

Table 1 Characteristics of the study patients at baseline stratified by eGFR. Values are presented as $n$ (\%) or means \pm s.D.s or median (range); Further analysis were done after LOG transition if the quantitative data does not conform to normal distribution.

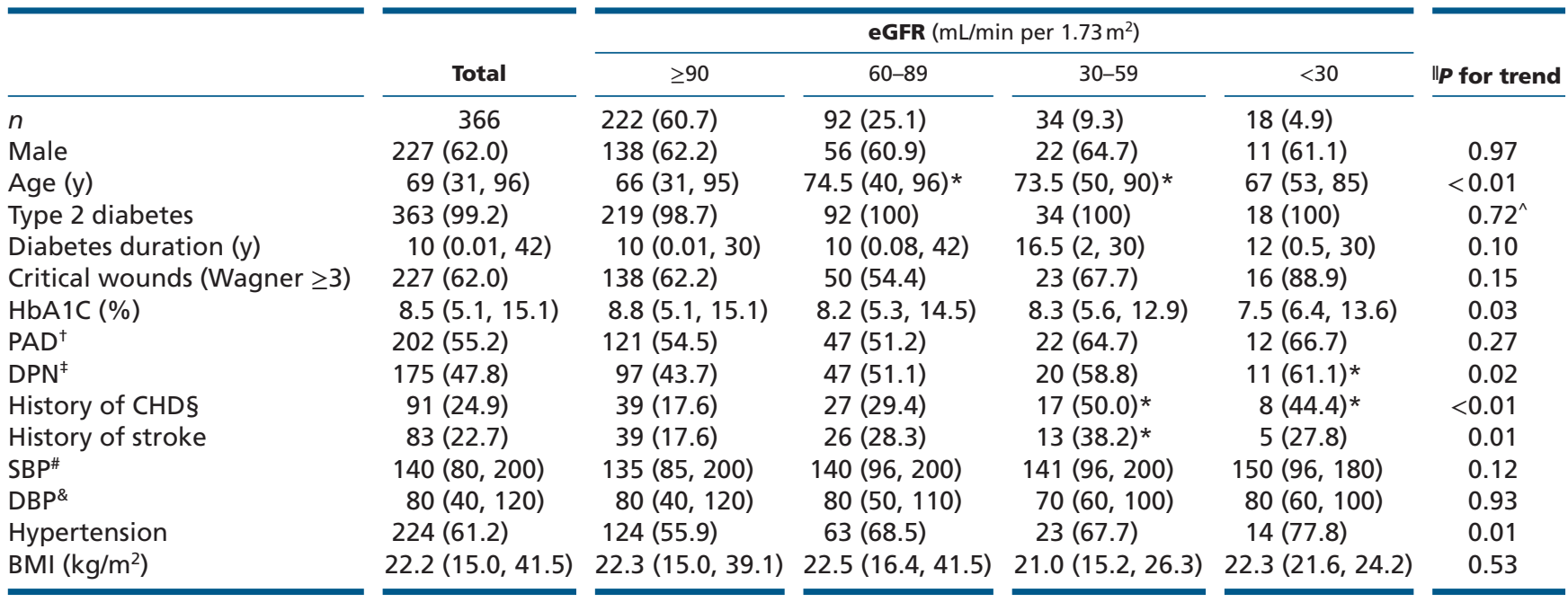

${ }^{*} P<0.05$ vs eGFR $\geq 90 \mathrm{~mL} / \mathrm{min} / 1.73 \mathrm{~m}^{2} ;{ }^{\dagger} \mathrm{PAD}$ : peripheral artery disease; ${ }^{\ddagger} \mathrm{DPN}$, diabetic peripheral neuropathy; ${ }^{\S} \mathrm{CHD}$, coronary heart disease; $11 P$ for trend through eGFR categories. "SBP, systolic blood pressure; DBP, diastolic blood pressure; ^ ${ }^{\wedge}$ Calculated from Fish exact probability. 
Table 2 Treatment at baseline Data are presented as $n(\%)$.

\begin{tabular}{|c|c|}
\hline & Total \\
\hline$n$ & 366 \\
\hline$\beta$-blocker & $14(3.8)$ \\
\hline ACEI/ARB & $147(40.2)$ \\
\hline Diuretics & $115(31.4)$ \\
\hline Antiplatelet & $186(50.8)$ \\
\hline Insulin use & $339(92.6)$ \\
\hline Dialysis & $6(1.8)$ \\
\hline History of revascularization & $14(3.8)$ \\
\hline
\end{tabular}

\begin{tabular}{c}
\hline$\geq 90$ \\
\hline $222(60.7)$ \\
$5(2.3)$ \\
$76(34.2)$ \\
$50(22.5)$ \\
$113(50.9)$ \\
$208(93.7)$ \\
0 \\
$9(4.05)$ \\
\hline
\end{tabular}

\begin{tabular}{|c|c|}
\hline \multicolumn{2}{|c|}{ eGFR $\left(\mathrm{mL} / \mathrm{min}\right.$ per $\left.1.73 \mathrm{~m}^{2}\right)$} \\
\hline $60-89$ & $30-59$ \\
\hline $92(25.1)$ & $34(9.3)$ \\
\hline $7(7.6)$ & 0 \\
\hline $44(47.8)$ & $18(52.9)$ \\
\hline $36(39.1) *$ & $20(58.8) *$ \\
\hline $44(47.8)$ & $18(52.9)$ \\
\hline $82(89.1)$ & $32(94.1)$ \\
\hline 0 & 0 \\
\hline $4(4.35)$ & $1(2.94)$ \\
\hline
\end{tabular}

\begin{tabular}{c}
$<30$ \\
\hline $18(4.9)$ \\
$2(11.1)$ \\
$9(50.0)$ \\
$9(50.0)$ \\
$11(61.1)$ \\
$17(94.4)$ \\
$6(33.33) *$ \\
0
\end{tabular}

$\| P$ for trend

$* P<0.05$ vs eGFR $\geq 90 \mathrm{~mL} / \mathrm{min}$ per $1.73 \mathrm{~m}^{2} ; \| P$ for trend through eGFR categories; ACEI, angiotensin converting enzyme inhibitors; ARB, angiotensin receptor blocker; History of revascularization: revascularization done in an other hospital before enrolment, which included balloon valvuloplasty, vascular Stent, vascular bypass and (or) blood vessel prosthesis.

The accumulated healing rates at 3, 6 and 12 months were $60.1,73.3$ and $90.0 \%$, respectively. The median time from initial treatment to healing was 2 months.

In the first six months, the healing rate of the normal group was $77.1 \%$ (145 out of 188 alive patients gained primary healing) whereas the rate was much lower in reduced eGFR group, with $64.7 \%$ (44/68), 45.5\% (10/22) and $30.8 \%(4 / 13)$ in the mildly, moderately and severely reduced group respectively ( $P<0.01$ in $\chi^{2}$ test, Fig. $\left.1 \mathrm{~A}\right)$.
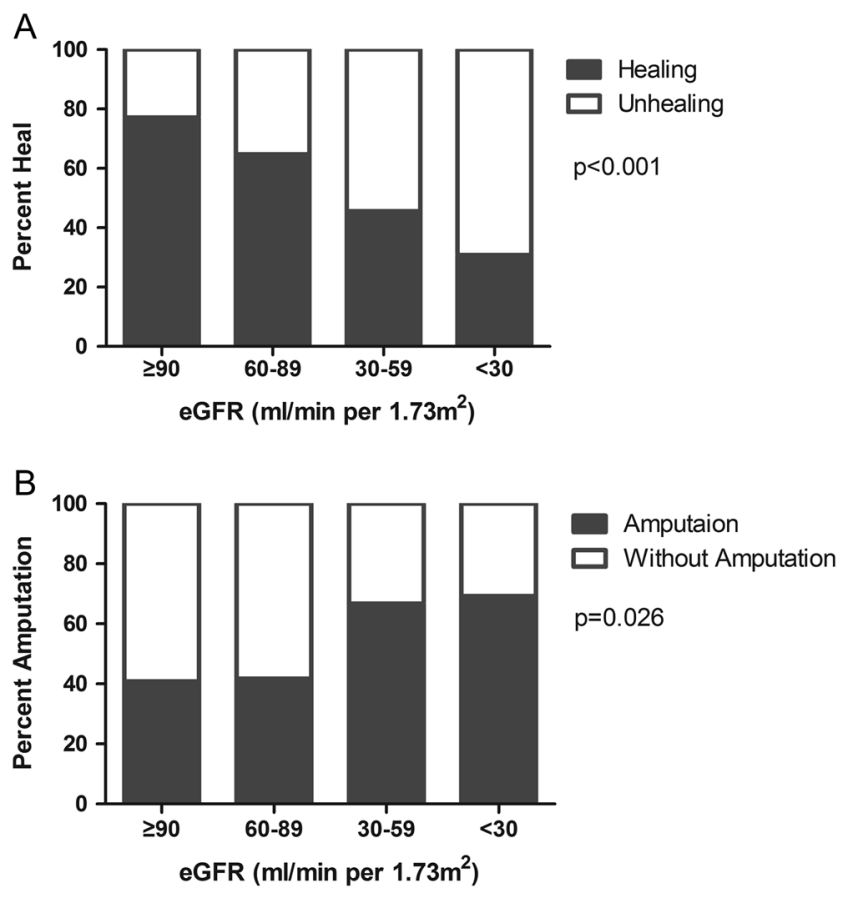

Figure 1

The comparisons of healing and amputation rate at 6 months between eGFR groups. (A) The healing rate at 6 months among eGFR groups. (B) The amputation rate at 6 months among eGFR groups. $\chi^{2}$ tests were performed to make comparisons among groups.
Similarly, the Log-rank test and Cox proportional-hazards models also showed that patients with reduced eGFR exhibited a higher prevalence of ulcer healing failure (Fig. 2A, Table 3), with hazard ratios of 2.13 (95\% CI: $1.18-3.83, P=0.01$ ) and 2.85 (95\% CI: $1.46-5.59, P=0.002$ ) for moderately and severely reduced eGFR groups, respectively. Further adjustments for age, sex, Wagner grade, DPN, PAD, HbA1c and treatment confirmed the results, with the risk of healing failure increased by $108 \%$ and $184 \%$ for the moderately and severely reduced groups respectively, compared with that of the normal group. A decreasing healing trend across eGFR categories was also evident in both univariate and multiple analyses (Table 3).

\section{Amputation}

During the follow-up, $48.6 \%(162 / 333)$ of patients underwent amputations, of which 146 had minor ones and 16 had major amputations. In the first six months, approximately $40 \%$ of patients in the normal $(40.8 \%$, 80 out of 196 alive patients) and mildly (41.7\%, 30/72) reduced eGFR groups underwent amputation, while the rate was above $60 \%$ in the moderately $(66.7 \%, 16 / 24)$ and severely $(69.2 \%, 9 / 13)$ reduced eGFR groups $(P=0.026$ in $\chi^{2}$ test, Fig. 1B). In COX regression models, those with moderately reduced eGFR had a nearly two-fold higher risk of total amputation than patients with normal eGFR, even after adjusting for age, sex, Wagner grade, DPN, and PAD (hazard ratios (HRs) were 1.96 and 1.70 in Model 1 and Model 3, respectively, Table 3). Further adjustment of HbA1c and treatment showed moderately reduced eGFR was marginally associated with higher risk of total amputation (Model 4, Table 3). For patients with severely reduced eGFR, although their amputation rate was higher than that in the normal group (Fig. 2B), the results from Cox proportional-hazards models failed to show 

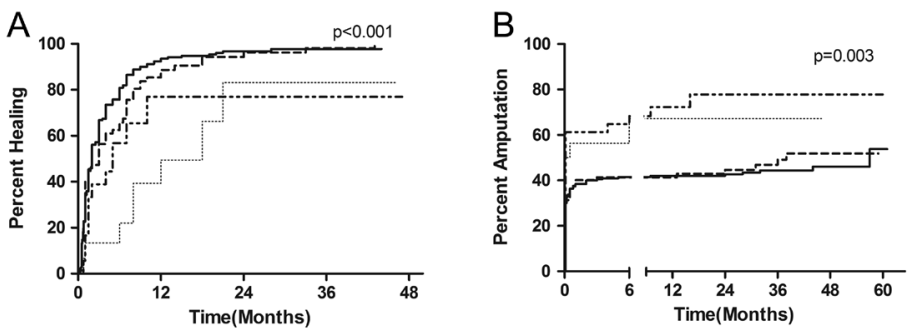

$$
\begin{aligned}
& \text { eGFR }\left(\mathrm{m} / / \mathrm{min} \text { per } 1.73 \mathrm{~m}^{2}\right) \\
& -\geq 90 \\
& --. \quad 60-89 \\
& -. \quad 30-59 \\
& -<30
\end{aligned}
$$
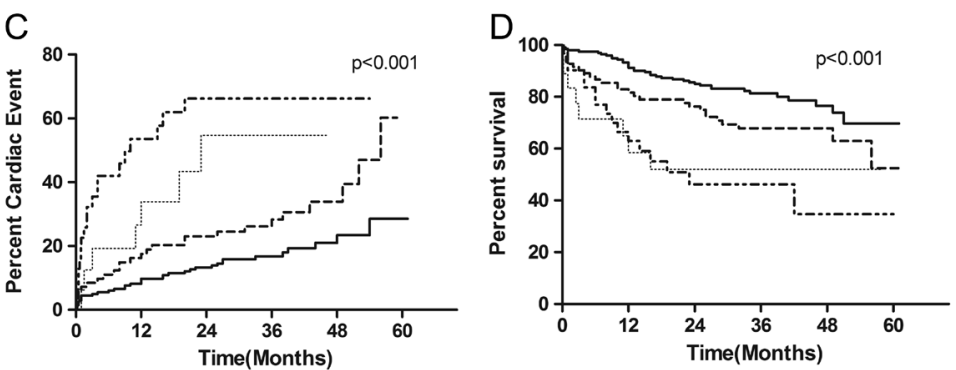

\section{Figure 2}

Kaplan-Meier curves of ulcer-related and patients-related outcomes stratified by eGFR groups. (A) The Kaplan-Meier curve of ucer healing in patients without major amputation among eGFR groups. (B) The Kaplan-Meier curve of total amputation among eGFR groups. (C) The KaplanMeier curve of cardiac event among eGFR groups. (D) The Kaplan-Meier curve of survival among eGFR groups. Log-rank tests were performed to make comparisons among groups. statistically higher risk for amputation (Table 3). The risk for major amputation was not significantly different among all four groups (all $P>0.05$, Table 3 ).

\section{Ulcer recurrence}

Re-emerging ulcers were observed in 144 (52.9\%) patients of the 272 patients who attained ulcer healing. The accumulated recurrence rates at 1, 2 and 3 years were
20.4, 35.5 and 47.1\%, respectively. Reduced eGFR did not

\begin{tabular}{|c|c|c|c|c|c|c|c|c|}
\hline \multirow[b]{3}{*}{ Outcome } & \multicolumn{7}{|c|}{ eGFR $\left(\mathrm{mL} / \mathrm{min}\right.$ per $\left.1.73 \mathrm{~m}^{2}\right)$} & \multirow[b]{3}{*}{$P$ trend } \\
\hline & \multirow[b]{2}{*}{$\geq 90(n=201)$} & \multicolumn{2}{|c|}{$60-89(n=83)$} & \multicolumn{2}{|l|}{$30-59(n=31)$} & \multicolumn{2}{|l|}{$<30(n=18)$} & \\
\hline & & $\mathrm{HR}(95 \% \mathrm{Cl})$ & $P$ & $\mathrm{HR}(95 \% \mathrm{Cl})$ & $P$ & $\mathrm{HR}(95 \% \mathrm{Cl})$ & $P$ & \\
\hline \multicolumn{9}{|c|}{ Healing failure } \\
\hline Model 1 & Ref & $1.20(0.90,1.60)$ & 0.20 & $2.13(1.18,3.83)$ & 0.01 & $2.85(1.46,5.59)$ & $<0.01$ & $<0.01$ \\
\hline Model 2 & Ref & $1.12(0.83,1.51)$ & 0.46 & $2.02(1.14,3.66)$ & 0.02 & $2.96(1.51,5.81)$ & $<0.01$ & $<0.01$ \\
\hline Model 3 & Ref & $1.20(0.88,1.64)$ & 0.24 & $2.13(1.17,3.89)$ & 0.01 & $2.60(1.32,5.13)$ & $<0.01$ & $<0.01$ \\
\hline Model 4 & Ref & $1.08(0.78,1.51)$ & 0.63 & $2.08(1.13,3.82)$ & 0.02 & $2.84(1.25,6.49)$ & 0.01 & $<0.01$ \\
\hline \multicolumn{9}{|c|}{ Total amputation } \\
\hline Model 1 & Ref & $1.07(0.73,1.56)$ & 0.73 & $1.96(1.23,3.10)$ & $<0.01$ & $1.63(0.87,3.06)$ & 0.13 & $<0.01$ \\
\hline Model 2 & Ref & $1.07(0.73,1.59)$ & 0.72 & $1.97(1.22,3.16)$ & $<0.01$ & $1.63(0.87,3.06)$ & 0.13 & 0.01 \\
\hline Model 3 & Ref & $1.15(0.77,1.70)$ & 0.50 & $1.70(1.06,2.73)$ & 0.03 & $1.00(0.53,1.89)$ & 0.99 & 0.24 \\
\hline Model 4 & Ref & $1.19(0.78,1.82)$ & 0.41 & $1.60(0.97,2.65)$ & 0.06 & $0.64(0.26,1.61)$ & 0.35 & 0.57 \\
\hline \multicolumn{9}{|c|}{ Major amputation } \\
\hline Model 1 & Ref & $1.21(0.36,4.02)$ & 0.76 & $1.79(0.38,8.47)$ & 0.46 & $3.20(0.68,15.12)$ & 0.14 & 0.15 \\
\hline Model 2 & Ref & $1.44(0.41,5.05)$ & 0.57 & $2.12(0.43,10.40)$ & 0.36 & $3.30(0.70,15.68)$ & 0.13 & 0.11 \\
\hline Model 3 & Ref & $1.82(0.49,6.69)$ & 0.37 & $2.22(0.45,11.08)$ & 0.33 & $2.20(0.44,10.87)$ & 0.33 & 0.21 \\
\hline Model 4 & Ref & $2.19(0.53,8.94)$ & 0.28 & $3.38(0.64,17.99)$ & 0.15 & $2.40(0.25,23.42)$ & 0.45 & 0.16 \\
\hline \multicolumn{9}{|l|}{ Recurrence } \\
\hline Model 1 & Ref & $1.00(0.66,1.52)$ & 0.99 & $0.62(0.25,1.54)$ & 0.31 & $0.72(0.23,2.29)$ & 0.58 & 0.37 \\
\hline Model 2 & Ref & $1.04(0.67,1.60)$ & 0.87 & $0.65(0.26,1.61)$ & 0.35 & $0.71(0.22,2.26)$ & 0.56 & 0.42 \\
\hline Model 3 & Ref & $1.07(0.69,1.65)$ & 0.77 & $0.66(0.26,1.64)$ & 0.37 & $0.74(0.23,2.36)$ & 0.61 & 0.49 \\
\hline Model 4 & Ref & $1.11(0.70,1.78)$ & 0.66 & $0.51(0.19,1.43)$ & 0.20 & $1.15(0.35,3.74)$ & 0.82 & 0.65 \\
\hline
\end{tabular}
significantly correlate with the recurrence of ulcers either in univariate or in multivariate analysis (All $P>0.05$, Table 3).

Table 3 Unadjusted and adjusted HRs $(95 \% \mathrm{Cl})$ of the association between eGFR and ulcer-related outcomes.

Model 1: univariate analysis; Model 2: adjusted for age, sex; Model 3: adjusted for age, sex, Wagner grade, DPN, PAD; Model 4: adjusted for age, sex, Wagner grade, DPN, PAD, HbA1c, history of revascularization, Dialysis, insulin use. 
failure, 57; acute coronary syndrome, 12 ; acute coronary syndrome combined with acute heart failure, 9; sudden cardiac death, 6), stroke in 42 and both occurred in 5 patients.

The accumulated rates of cardiac events at 1,2 and 3 years were $16.9 \%, 22.3 \%$ and $27.5 \%$ respectively. Over $50 \%$ of the patients with reduced eGFR suffered cardiac events, whereas only less than $30 \%$ of patients in the normal eGFR group had cardiac events (Fig. 2C). Of note, even those patients in the mildly reduced eGFR group had a nearly two-fold higher risk of cardiac events than those in the normal group (HR=1.88, 95\% CI: 1.13-3.13), though further adjustments attenuated the results (Table 4). Moderately reduced eGFR was an independent predictor of cardiac events with HRs of 5.25 in the multivariate regression model. Severely reduced eGFR was associated with higher incidence of cardiac events independent of age, sex, Wagner grade and other diabetic complications, but the result was attenuated by further adjustment of HbA1c and treatment (Table 4).

A total of 47 participants were observed to have one or more cerebrovascular events. The accumulated incidences at 1, 2 and 3 years were $8.8 \%, 13.3 \%$ and $15.9 \%$ respectively. Even in the patients with normal eGFR, over $15 \%$ of participants had a stroke during the 3-year follow-up period. However, only five of the total 33 patients in the moderate group, and two of 18 patients in the severe group suffered a stroke. In the COX regression model, there was no significant correlation between the occurrence of cerebrovascular events and eGFR (all $P>0.05$, Table 4).

\section{Mortality}

Eighty-nine (26.7\%) of the participants died during this study, including 44 (13.2\%) from cardiovascular disease (32 from acute heart failure, 6 from sudden cardiac death, and 6 from myocardial infarction), 10 (3.0\%) from stroke, 9 (2.7\%) from renal failure, 8 (2.4\%) from infection, $3(0.9 \%)$ from malignancies, and $6(1.8 \%)$ from other causes. The exact causes of the remaining $9(2.7 \%)$ deaths were unknown, because these patients died at home without the presence of doctors and (or) an autopsy. The survival rates at 1,3 and 5 years were $84.5 \%, 73.0 \%$ and $60.8 \%$, respectively.

EGFR was a positive predictor for survival (Fig. 2D, Table 4), with HRs that increased from 1.78 (95\% CI: $1.09-2.91, P=0.02)$ in the mildly reduced eGFR group to 3.78 (95\% CI: $1.76-8.12, P=0.0007)$ in the severely reduced eGFR group. After adjustment by the complicated confounders and treatment variables, moderately and severely reduced eGFR were found to be independently associated with mortality, with risks of death three and four times higher than those in the normal eGFR group (Table 4). Nonetheless, this correlation was not observed in the mildly reduced eGFR group after adjustment (HR: $1.49,95 \%$ CI: 0.66-3.34, $P=0.34$, Table 4).

Table 4 Unadjusted and adjusted HRs $(95 \% \mathrm{Cl})$ of the association between eGFR and patients-related outcomes.

\begin{tabular}{|c|c|c|c|c|c|c|c|c|}
\hline \multirow[b]{3}{*}{ Outcome } & \multicolumn{7}{|c|}{ eGFR $\left(\mathrm{mL} / \mathrm{min}\right.$ per $\left.1.73 \mathrm{~m}^{2}\right)$} & \multirow[b]{3}{*}{$P$ trend } \\
\hline & \multirow[b]{2}{*}{$\geq 90(n=201)$} & \multicolumn{2}{|c|}{$60-89(n=83)$} & \multicolumn{2}{|c|}{$30-59(n=31)$} & \multicolumn{2}{|c|}{$<30(n=18)$} & \\
\hline & & $\mathrm{HR}(95 \% \mathrm{Cl})$ & $P$ & $\mathrm{HR}(95 \% \mathrm{Cl})$ & $P$ & $\mathrm{HR}(95 \% \mathrm{Cl})$ & $P$ & \\
\hline \multicolumn{9}{|c|}{ Cardiac event } \\
\hline Model 1 & Ref & $1.88(1.13-3.13)$ & 0.02 & $6.43(3.64-11.34)$ & $<0.01$ & $3.80(1.68-8.62)$ & $<0.01$ & $<0.01$ \\
\hline Model 2 & Ref & $1.43(0.84-2.43)$ & 0.19 & $5.12(2.87-9.15)$ & $<0.01$ & 3.77 (1.66-8.55) & $<0.01$ & $<0.01$ \\
\hline Model 3 & Ref & $1.31(0.75-2.29)$ & 0.34 & 3.77 (2.01-7.05) & $<0.01$ & $2.51(1.09-5.80)$ & 0.03 & $<0.01$ \\
\hline Model 4 & Ref & $1.55(0.69-3.52)$ & 0.29 & $5.25(2.17-12.89)$ & $<0.01$ & $2.99(0.89-10.09)$ & 0.08 & $<0.01$ \\
\hline \multicolumn{9}{|c|}{ Cerebrovascular event } \\
\hline Model 1 & Ref & $1.20(0.62-2.32)$ & 0.60 & $1.82(0.70-4.74)$ & 0.22 & $1.24(0.29-5.21)$ & 0.77 & 0.32 \\
\hline Model 2 & Ref & $0.93(0.47-1.87)$ & 0.85 & $1.50(0.57-3.97)$ & 0.41 & $1.17(0.28-4.96)$ & 0.83 & 0.61 \\
\hline Model 3 & Ref & $0.78(0.37-1.64)$ & 0.52 & $1.51(0.52-4.36)$ & 0.45 & $1.25(0.28-5.56)$ & 0.77 & 0.73 \\
\hline Model 4 & Ref & $0.63(0.17-2.30)$ & 0.49 & $1.93(0.41-9.16)$ & 0.41 & $0.89(0.07-12.32)$ & 0.93 & 0.79 \\
\hline \multicolumn{9}{|l|}{ Death } \\
\hline Model 1 & Ref & $1.78(1.09-2.91)$ & 0.02 & $3.98(2.21-7.16)$ & $<0.01$ & $3.78(1.76-8.12)$ & $<0.01$ & $<0.01$ \\
\hline Model 2 & Ref & $1.20(0.72-1.99)$ & 0.49 & $2.88(1.58-5.23)$ & $<0.01$ & $3.61(1.68-7.78)$ & $<0.01$ & $<0.01$ \\
\hline Model 3 & Ref & $1.13(0.66-1.93)$ & 0.67 & $2.24(1.15-4.38)$ & 0.02 & $3.27(1.47-7.27)$ & $<0.01$ & $<0.01$ \\
\hline Model 4 & Ref & $1.49(0.66-3.34)$ & 0.34 & 3.54 (1.36-9.20) & 0.01 & $4.45(1.23-16.07)$ & 0.02 & $<0.01$ \\
\hline
\end{tabular}

Model 1: univariate analysis; Model 2: adjusted for age, and sex; Model 3: adjusted for age, sex, Wagner grade, DPN, PAD, CHD, Stroke, SBP, and diabetes duration; Model 4: adjusted for age, sex, Wagner grade, DPN, PAD, CHD, Stroke, SBP, diabetes duration, HbA1C, revascularization, dialysis, insulin use, antiplatelet, ACEI/ARB, diuretic. 


\section{Discussion}

In this cohort study, we found that a moderate or severe decrease in eGFR was an independent predictor of poor outcomes in DFU patients, with higher rates of healing failure, a higher risk of cardiac events(significant in moderately group and marginally significant in the severely one), and higher all-cause mortality compared with the normal group. Meanwhile, moderately reduced eGFR was marginally associated with an increased need for amputation. Patients with mildly reduced eGFR exhibited a higher prevalence of cardiac events and death in univariate analysis, although no significant correlation was shown with ulcer-related and patient-related outcomes in multivariate analysis.

This study clarified how different levels of decreased eGFR impacted the foot, cardiovascular system and survival when a foot ulcer has already occurred in patients with diabetes. These results might help doctors realize the detrimental role of renal insufficiency in the progress of DFU even with proper foot treatment.

Renal insufficiency, regardless of whether it is defined as eGFR $<60 \mathrm{~mL} / \mathrm{min}$ per $1.73 \mathrm{~m}^{2}(3,18)$ or as serum creatinine above $1.5 \mathrm{mg} / \mathrm{dL}(19,20)$, has been reported to affect ulcer healing in patients with diabetes. Our stratified analysis reconfirmed these results and further illustrated no statistically significant impact of mildly reduced eGFR on ulcer healing. Wound healing, included the phases of inflammation, proliferation and remodeling, is a complex process that requires a well-organized immune response, adequate blood or nutrition supply and normal innervation (21). Immune dysregulation in patients with renal insufficiency depletes the defences against infection and delays ulcer healing (22). Meanwhile, chronic kidney disease is commonly complicated with PAD and uremic neuropathy, which harm the process of wound healing $(23,24)$. Hypoalbuminemia, anemia and edema caused by renal failure may also explain the effect of moderately or severely decreased eGFR on poor ulcer healing in DFU $(25,26,27)$.

Attention has been drawn to the remarkably high rate of amputations, and particularly major amputations, in patients with diabetes on either dialysis $(7,28,29,30)$ or with renal insufficiency $(7,31,32)$. The present study found that the adverse impact of moderately reduced eGFR on amputation was marginally significant in DFU patients. However, the results from our study did not support the inclusion of eGFR $<30 \mathrm{~mL} / \mathrm{min}$ per $1.73 \mathrm{~m}^{2}$ as a risk factor. The likely explanation might be our subjective determination of amputation. In our clinical practice, indications for amputation in patients with eGFR of $<30 \mathrm{~mL} / \mathrm{min}$ or dialysis were handled strictly, because these patients had relatively higher rates of postoperative complications or mortality $(33,34)$ and lower rates of wound healing after amputation (3). Therefore, amputations were performed only in patients with lifethreatening ulcers. Other patients lived with ulcers that were carefully protected from infection. Therefore, the subjective determination of amputation might cover the true association between severely reduced eGFR and the need for amputation. For major amputations, the results were similar to those of total amputation but did not reach statistical significance. This result might be due to the limited number of major amputations (only 16) performed in our study.

A limited number of studies have concentrated on ulcer recurrence and its risk factors (35). Although Waaijman $\mathrm{R}$ carefully discussed the re-emerging ulcers in 171 patients with diabetes with recently healed ulcers, the impact of renal function was not mentioned in that study (36). Data from several other published studies did not support the correlation between renal insufficiency and recurrence. Connor \& Mahdi reported that serum creatinine and proteinuria were similar between those who have a high risk of repetitive ulceration and their low risk counterparts (37). In another 3-year follow-up study on 79 patients with diabetes with healed ulcers, the proportion of end-stage renal disease was not markedly different in patients with and without recurrence. Furthermore, no renal-related index could be selected into the multivariate stepwise logistic regression model for re-ulceration (38). Consistent with these published data, our stratified study found none of the reduced eGFR groups to be associated with recurrence. Based on the discussion above regarding the detrimental role of renal insufficiency on blood supply, nutrition status, innervation and immune response, one may speculate that reduced eGFR is also a risk factor for re-emerging ulcers. However, the results were negative both in other studies and in ours; the reasons remain unknown. One likely explanation is that most of the studies were conducted in diabetes or foot care centers, where proper treatment for blood supply and peripheral nerves were given both in the hospital and during the follow-up period once a foot ulcer occurred, and therefore partially offsetting the adverse role of renal insufficiency in the re-emergence of foot ulcers. Another reason might be the decrease in the ability, intensity and duration of patients' activity with the deterioration of renal function, which, to some extent, protected patients from excessive plantar pressure and trauma. 
Unlike amputation and mortality, cardiac events have rarely been considered important endpoints in follow-up studies of DFU. Indeed, diabetic foot disease has been observed to be a marker for cardiac events in recent decades (39). Our previous study (40) and several other reports $(41,42,43)$ observed that patients with diabetes with foot ulcers were more likely to be complicated with cardiac disease and had a higher incidence of new cardiac events than those without foot ulcers. Additionally, cardiovascular events were suggested to be the leading cause of death in most long-term follow-up studies of DFU $(3,7)$, and the mortality of DFU might be decreased by aggressive cardiovascular risk management (44). Therefore, we regarded cardiac events as one of the observed endpoints in our study. Data from our study once again supported the high incidence of cardiac events in DFU, since over $20 \%$ of DFU patients experienced one or more cardiac events, even in the normal eGFR group. Moderately and severely reduced eGFR, which were previously proven to be independent predictors for cardiac events in patients with diabetes $(45,46)$, made matters worse in DFU, as the relative risk was increased five times higher (in the moderate group) and three times higher (in the severe group) than those with normal eGFR.

The independent impact of mildly reduced eGFR on cardiovascular disease remains unclear $(47,48)$. Wang found that patients with diabetes with eGFR of $60-89(\mathrm{~mL} /$ min per $1.73 \mathrm{~m}^{2}$ ) were at a significantly higher risk of coronary heart disease and stroke in a follow-up study containing 11940 Caucasian and 16451 AfricanAmerican patients with diabetes (49). However, in another study of 6233 subjects in the US general population during an average of 15 years of follow-up, the association between mild renal insufficiency and cardiovascular disease was not independent (50). In our present study, mildly decreased eGFR was found to be associated with a higher risk of cardiac events in univariate analysis. However, this association seemed to be attributed to the concurrence of critical ulcers, PAD, DPN, cardiovascular disease, stroke and hypertension in the mildly decreased eGFR group at baseline.

Conflicting results regarding the impact of eGFR on stroke were reported in previous studies in both the general population $(51,52)$ and in patients with diabetes $(53,54)$. Little was previously known about this association in patients with DFU. In the present study, whether adjusted or not, eGFR was not significantly associated with cerebrovascular events in DFU patients. However, the limited number of strokes observed in the moderately and severely reduced eGFR groups might impact the accuracy of this statistic. Thus, the divergent impact of eGFR on cardiac events and cerebrovascular events, which share many risk factors in common, found in our study remains to be ascertained, and the underlying mechanism needs further investigation.

It is well established that foot ulcers increase the risk of mortality in patients with diabetes (55). Data from our present study showed that renal insufficiency may increase this risk further still, with progressively increased HRs for all-cause mortality in DFU patients from the onset of renal impairment to end-stage renal disease. Nevertheless, the correlation between mildly reduced eGFR and mortality was not independent. Older age and the co-occurrence of more cardiovascular disease, stroke and critical foot ulcers at baseline seemed to be the confounders between mildly reduced eGFR and increased risk of death. Consistent with our results, a previous 6.5 year follow-up study in $84 \mathrm{DFU}$ patients reported creatinine clearance $\leq 60 \mathrm{~mL} / \mathrm{min}$ to be an independent predictor for mortality (3). Similarly, serum creatinine concentration $\geq 1.5 \mathrm{mg} / \mathrm{dL}$ was found to be associated with mortality in another study of 247 DFU patients (7). These findings from our study and other published articles provide information on strategies that may further attenuate mortality in this group of patients.

There were several limitations in our study. First, the study cohort was recruited in one hospital, inevitably including selection bias. Second, the association between eGFR and specific-mortality was not analysed, because that incidence of subdivided death was relatively low and might cause insufficient statistical power. Finally, 33 (9.0\%) patients were lost from the cohort. However, the rate of drop-out was low, and the baseline characteristics were similar between the total and drop-out patients. Therefore, the impact of this factor on the overall result is minimal and will not change the conclusion.

In conclusion, moderately and severely reduced eGFR is closely associated with unfavorable outcomes in patients with DFU. This study indicates that renal protection is crucial in the management of diabetic foot ulcers.

\section{Declaration of Interest}

The authors declare that there is no conflict of interest that could be perceived as prejudicing the impartiality of this study.

\section{Funding}

This study was supported by National Natural Science Foundation of China (grant number 81370977). 


\section{Author contribution statement}

Zhengyi Tang designed the study, reviewed the data and manuscript and was the guarantor of the work. Yang He researched data, performed statistical analysis and wrote the manuscript. Hongjie Qian, Lei Xu and Shanshan Zhang researched data and performed statistical analysis. Xueming Gu, Junyi Gu, Jianyuan Shi and Yaping Shen researched data. Jianmin Liu reviewed the manuscript.

\section{Acknowledgements}

The authors thank all 366 patients for their valuable contribution to the study. The authors also thank all the physicians, nurses and technicians involved for their assistance.

\section{References}

1 Boulton AJ, Vileikyte L, Ragnarson-Tennvall G \& Apelqvist J. The global burden of diabetic foot disease. Lancet 2005366 1719-1724. (doi:10.1016/s0140-6736(05)67698-2)

2 Apelqvist J, Larsson J \& Agardh CD. Long-term prognosis for diabetic patients with foot ulcers. Journal of Internal Medicine 1993233 485-491. (doi:10.1111/j.1365-2796.1993.tb01003.x)

3 Ghanassia E, Villon L, Thuan Dit Dieudonné JF, Boegner C, Avignon A \& Sultan A. Long-term outcome and disability of diabetic patients hospitalized for diabetic foot ulcers: a 6.5-year follow-up study. Diabetes Care 200831 1288-1295. (doi:10.2337/dc07-2145)

4 Wolf G, Müller N, Busch M, Eidner G, Kloos C, Hunger-Battefeld W $\&$ Müller UA. Diabetic foot syndrome and renal function in type 1 and 2 diabetes mellitus show close association. Nephrology, Dialysis, Transplantation 200924 1896-1901. (doi:10.1093/ndt/gfn724)

5 Pollard J, Hamilton GA, Rush SM \& Ford LA. Mortality and morbidity after transmetatarsal amputation: retrospective review of 101 cases. Journal of Foot \& Ankle Surgery 200645 91-97. (doi:10.1053/j. jfas.2005.12.011)

6 Miyajima S, Shirai A, Yamamoto S, Okada N \& Matsushita T. Risk factors for major limb amputations in diabetic foot gangrene patients. Diabetes Research and Clinical Practice 20067 272-279. (doi:10.1016/j. diabres.2005.07.005)

7 Morbach S, Furchert H, Gröblinghoff U, Hoffmeier H, Kersten K, Klauke GT, Klemp U, Roden T, Icks A, Haastert B et al. Long-term prognosis of diabetic foot patients and their limbs: amputation and death over the course of a decade. Diabetes Care 201235 2021-2027. (doi:10.2337/dc12-0200)

8 Levey AS, Perrone RD \& Madias NE. Serum creatinine and renal function. Annual Review of Medicine 198839 465-490. (doi:10.1146/ annurev.me.39.020188.002341)

9 Froissart M, Rossert J, Jacquot C, Paillard M \& Houillier P. Predictive performance of the modification of diet in renal disease and Cockcroft-Gault equations for estimating renal function. Journals of the American Society of Nephrology 200516 763-773. (doi:10.1681/ asn.2004070549)

10 Apelqvist J, Bakker K, van Houtum WH \& Schaper NC. International Working Group on the Diabetic Foot (IWGDF) Editorial Board. Practical guidelines on the management and prevention of the diabetic foot: based upon the InternationalConsensus on the Diabetic Foot (2007) Prepared by the International Working Group on the Diabetic Foot. Diabetes Metabolism: Research and Reviews 200824 (Supplement 1) S181-S187. (doi:10.1002/dmrr.848)

11 Lipsky BA, Peters EJ, Berendt AR, Senneville E, Bakker K, Embil JM, Lavery LA, Urbančič-Rovan V \& Jeffcoate WJ. International Working Group on Diabetic Foot. Specific guidelines for the treatment of diabetic foot infections 2011. Diabetes Metabolism: Research and Reviews 201228 (Supplement 1) 234-235. (doi:10.1002/dmrr.2251)
12 Schaper NC, Andros G, Apelqvist J, Bakker K, Lammer J, Lepantalo M, Mills JL, Reekers J, Shearman CP, Zierler RE et al. International Working Group on Diabetic foot. Specific guidelines for the diagnosis and treatment of peripheral arterial disease in a patients with diabetic ulceration of the foot 2011. Diabetes Metabolism: Research and Reviews 201228 (Supplement 1) 236-237. (doi:10.1002/dmrr.2252)

13 Ma YC, Zuo L, Chen JH, Luo Q, Yu XQ, Li Y, Xu JS, Huang SM, Wang LN, Huang $\mathrm{W}$ et al. Modified glomerular filtration rate estimating equation for Chinese patients with chronic kidney disease. Journals of the American Society of Nephrology 200617 2937-2944. (doi:10.1681/ASN.2006040368)

14 Apelqvist J, Bakker K, van Houtum WH, Nabuurs-Franssen MH \& Schaper NC. International consensus and practical guidelines on the management and the prevention of the diabetic foot: International Working Group on the Diabetic Foot. Diabetes Metabolism: Research and Reviews 200016 (Supplement 1) S84-S92. (doi:10.1002/15207560(200009/10)16:1+<::AID-DMRR113>3.0.CO;2-S)

15 Wagner FW Jr. The dysvascular foot: a system for diagnosis and treatment. Foot Ankle 19812 64-122. (doi:10.1177/10711007810020 0202)

16 Boulton AJ, Vinik AI, Arezzo JC, Bril V, Feldman EL, Freeman R, Malik RA, Maser RE, Sosenko JM \& Ziegler D. Diabetic neuropathies: a statement by the American Diabetes Association. Diabetes Care 2005 28 956-962 (doi:10.2337/diacare.28.4.956)

17 Morbach S, Lutale JK, Viswanathan V, Möllenberg J, Ochs HR, Rajashekar S, Ramachandran A \& Abbas ZG. Regional differences in risk factors and clinical presentation of diabetic foot lesions. Diabetic Medicine 200421 91-95. (doi:10.1046/j.1464-5491.2003.01069.x)

18 Zubair M, Malik A \& Ahmad J. The impact of creatinine clearance on the outcome of diabetic foot ulcers in north Indian tertiary care hospital. Diabetology \& Metabolic Syndrome 20115 120-125. (doi:10.1016/j.dsx.2012.02.028)

19 Apelqvist J, Elgzyri T, Larsson J, Löndahl M, Nyberg P \& Thörne J. Factors related to outcome of neuroischemic/ischemic foot ulcer in diabetic patients. Journal of Vascular Surgery 201153 1582-1588. (doi:10.1016/j.jvs.2011.02.006)

20 Elgzyri T, Larsson J, Thörne J, Eriksson KF \& Apelqvist J. Outcome of ischemic foot ulcer in diabetic patents who had no invasive vascular intervention. European Journal of Vascular and Endovascular Surgery 201346 110-117. (doi:10.1016/j.ejvs.2013.04.013)

21 Lewis S, Raj D \& Guzman NJ. Renal failure: implications of chronic kidney disease in the management of the diabetic foot. Seminars in Vascular Surgery 201225 82-88. (doi:10.1053/j.semvascsurg.2012.04.007)

22 Dayton KD \& Lancaster LE. Part 2: Effects of renal failure and its treatment on the immune system and assessment of immune system function. ANNA Journal 199522 530-7572.

23 Garimella PS, Hart PD, O’Hare A, DeLoach S, Herzog CA \& Hirsch AT. Peripheral artery disease and CKD: a focus on peripheral artery disease as a critical component of CKD care. American Journal of Kidney Diseases 201260 641-654 (doi:10.1053/j.ajkd.2012.02.340)

24 Nielsen VK. The peripheral nerve function in chronic renal failure. VI. The relationship between sensory and motornerve conduction and kidney function, azotemia, age, sex and clinical neuropathy. Acta Medica Scandinavica 1973194 455-462 (doi:10.1111/j.0954-6820.1973.tb19471.x)

25 Hinchliffe RJ, Kirk B, Bhattacharjee D, Roe S, Jeffcoate W \& Game F. The effect of haemodialysis on transcutaneous oxygen tension in patients with diabetes-a pilot study. Nephrology, Dialysis, Transplantation 20062 1981-1983. (doi:10.1093/ndt/gfl241)

26 Chao CY, Zheng YP \& Cheing GL. The association between skin blood flow and edema on epidermal thickness in the diabetic foot. Diabetes Technology \& Therapeutics 201214 602-609. (doi:10.1089/ dia.2011.0301)

27 Chuan F, Zhang M, Yao Y, Tian W, He X \& Zhou B. Anemia in Patients With Diabetic Foot Ulcer: Prevalence, Clinical Characteristics, and Outcome. International Journal of Lower Extremity Wounds $2016 \mathbf{1 5}$ 220-226. (doi:10.1177/1534734616660224) 
28 Miyajima S, Shirai A, Yamamoto S, Okada N \& Matsushita T. Risk factors for major limb amputations in diabetic foot gangrene patients. Diabetes Research and Clinical Practice 20067 272-279. (doi:10.1016/j. diabres.2005.07.005)

29 Game FL, Chipchase SY, Hubbard R, Burden RP \& Jeffcoate WJ. Temporal association between the incidence of foot ulceration and the start of dialysis in diabetes mellitus. Nephrology, Dialysis, Transplantation 200621 3207-3210. (doi:10.1093/ndt/gfl427)

30 Quarantiello A, Lupattelli T \& Morabito A. Long-term prognosis of diabetic patients with critical limb ischemia: a population-based cohort study. Diabetes Care 200932 822-827. (doi:10.2337/dc08-1223)

31 Margolis DJ, Hofstad O \& Feldman HI. Association between renal failure and foot ulcer or lower-extremity amputation in patients with diabetes. Diabetes Care 200831 1331-1336. (doi:10.2337/dc07-2244)

32 Zubair M, Malik A \& Ahmad J. Incidence, risk factors for amputation among patients with diabetic foot ulcer in a North Indian tertiary care hospital. Foot 201222 24-30. (doi:10.1016/j.foot.2011.09.003)

33 O'Hare AM, Feinglass J, Reiber GE, Rodriguez RA, Daley J, Khuri S, Henderson WG \& Johansen KL. Postoperative mortality after nontraumatic lower extremity amputation in patients with renal insufficiency. Journals of the American Society of Nephrology 200415 427-434. (doi: 10.1097/01.ASN.0000105992.18297.63)

34 Belmont PJ Jr, Davey S, Orr JD, Ochoa LM, Bader JO \& Schoenfeld AJ. Risk factors for 30-day postoperative complications and mortality after below-knee amputation: a study of 2,911 patients from the national surgical quality improvement program. Journal of the American College of Surgeons 2011213 370-378. (doi:10.1016/j.jamcollsurg.2011.05.019)

35 Bus SA \& van Netten JJ. A shift inpriority in diabetic foot care and research: $75 \%$ of foot ulcers are preventable. Diabetes Metabolism: Research and Reviews 201632 (Supplement 1) 195-200. (doi:10.1002/dmrr.2738)

36 Waaijman R, de Haart M, Arts ML, Wever D, Verlouw AJ, Nollet F \& Bus SA. Risk factors for plantar foot ulcer recurrence in neuropathic diabetic patients. Diabetes Care 201437 1697-1705. (doi:10.2337/dc13-2470)

37 Connor H \& Mahdi OZ. Repetitive ulceration in neuropathic patients. Diabetes Metabolism: Research and Reviews 200420 (Supplement 1) S23-S28. (doi:10.1002/dmrr.446)

38 Dubský M, Jirkovská A, Bem R, Fejfarová V, Skibová J, Schaper NC \& Lipsky BA. Risk factors for recurrence of diabetic foot ulcers: prospective follow-up analysis in the Eurodiale subgroup. International Wound Journal 201310 555-561. (doi:10.1111/j.1742-481x.2012.01022.x)

39 Tuttolomondo A, Maida C \& Pinto A. Diabetic foot syndrome as a possible cardiovascular marker in diabetic patients. Journal of Diabetes Research 20152015268390.

40 Xu L, Qian H, Gu J, Shi J, Gu X \& Tang Z. Heart failure in hospitalized patients with diabetic foot ulcers: clinical characteristics and their relationship with prognosis. Journal of Diabetes 20135 429-438. (doi:10.1111/1753-0407.12062)

41 Chammas NK, Hill RL, Foster AV \& Edmonds ME. Is neuropathic ulceration the key to understanding increased mortality due to is chaemic heart disease in diabetic foot ulcer patients? A population approach using a proportionate model. Journal of International Medical Research 200230 553-559. (doi:10.1177/147323000203000602)

42 Löndahl M, Katzman P, Fredholm O, Nilsson A \& Apelqvist J. Is chronic diabetic foot ulcer an indicator of cardiac disease? Journal of Wound Care 200817 12-16 . (doi:10.12968/jowc.2008.17.1.27915)

43 Pinto A, Tuttolomondo A, Di Raimondo D, Fernandez P, La Placa S, Di Gati M \& Licata G. Cardiovascular risk profile and morbidity in subjects affected by type 2 diabetes mellitus with and without diabetic foot. Metabolism 20085 676-682. (doi:10.1016/j.metabol.2008.01.004)

44 Young MJ, McCardle JE, Randall LE \& Barclay JI. Improved survival of diabetic foot ulcer patients 1995-2008: possible impact of aggressive cardiovascular risk management. Diabetes Care 200831 2143-2147. (doi:10.2337/dc08-1242)

45 Ninomiya T, Perkovic V, de Galan BE, Zoungas S, Pillai A, Jardine M, Patel A, Cass A, Neal B, Poulter N et al. Albuminuria and kidney function independently predict cardiovascular and renal outcomes in diabetes. Journal of the American Society of Nephrology 200920 1813-1821. (doi:10.1681/ASN.2008121270)

46 Drury PL, Ting R, Zannino D, Ehnholm C, Flack J, Whiting M, Fassett R, Ansquer JC, Dixon P, Davis TM et al. Estimated glomerular filtration rate and albuminuria are independent predictors of cardiovascular events and death in type 2 diabetes mellitus: the Fenofibrate Intervention and Event Lowering in Diabetes (FIELD) study. Diabetologia 201154 32-43. (doi:10.1007/s00125-010-1854-1)

47 Henry RM, Kostense PJ, Bos G, Dekker JM, Nijpels G, Heine RJ, Bouter LM \& Stehouwer CD. Mild renal insufficiency is associated with increased cardiovascular mortality: the Hoorn Study. Kidney International 200262 1402-1407. (doi:10.1111/j.1523-1755.2002.kid571.x)

48 Chronic Kidney Disease Prognosis C, Matsushita K, van der Velde M, Astor BC, Woodward M, Levey AS, de Jong PE, Coresh J \& Gansevoort RT. Association of estimated glomerular filtration rate and albuminuria with all - cause and cardiovascular mortality in general population cohorts: a collaborative meta - analysis. Lancet 2010375 2073-2081. (doi:10.1016/S0140-6736(10)60674-5)

49 Wang Y, Katzmarzyk PT, Horswell R, Zhao W, Johnson J \& Hu G. Kidney function and the risk of cardiovascular disease in patients with type 2 diabetes. Kidney International 201485 1192-1199. (doi:10.1038/ki.2013.396)

50 Sarnak MJ, Levey AS, Schoolwerth AC, Coresh J, Culleton B, Hamm LL, McCullough PA, Kasiske BL, Kelepouris E, Klag MJ et al. American Heart Association Councils on Kidney in Cardiovascular Disease HBPRCC, Epidemiology, Prevention . Kidney disease as a risk factor for development of cardiovascular disease: a statement from the American Heart Association Councils on Kidney in Cardiovascular Disease, High Blood Pressure Research, Clinical Cardiology, and Epidemiology and Prevention. Circulation 2003108 2154-2169. (doi:10.1161/01.CIR.0000095676.90936.80)

51 Weiner DE, Tighiouart H, Amin MG, Stark PC, MacLeod B, Griffith JL, Salem DN, Levey AS \& Sarnak MJ. Chronic kidney disease as a risk factor for cardiovascular disease and all-cause mortality: a pooled analysis of community-based studies. Journals of the American Society of Nephrology 200415 1307-1315. (doi:10.1097/01. ASN.0000123691.46138.E2)

52 Nickolas TL, Khatri M, Boden-Albala B, Kiryluk K, Luo X, GervasiFranklin P, Paik M \& Sacco RL. The association between kidney disease and cardiovascular risk in a multiethnic cohort: findings from the Northern Manhattan Study (NOMAS). Stroke 200839 2876-2879. (doi:10.1161/STROKEAHA.107.513713)

53 Bouchi R, Babazono T, Yoshida N, Nyumura I, Toya K, Hayashi T, Hanai K, Tanaka N, Ishii A \& Iwamoto Y. Association of albuminuria and reduced estimated glomerular filtration rate with incident stroke and coronary artery disease in patients with type 2 diabetes. Hypertension Research 201033 1298-1304. (doi:10.1038/hr.2010.170)

54 So WY, Kong AP, Ma RC, Ozaki R, Szeto CC, Chan NN, Ng V, Ho CS, Lam CW, Chow CC et al. Glomerular filtration rate, cardiorenal end points, and all-cause mortality in type 2 diabetic patients. Diabetes Care 200629 2046-2052. (doi:10.2337/dc06-0248)

55 Brownrigg JR, Davey J, Holt PJ, Davis WA, Thompson MM, Ray KK \& Hinchliffe RJ. The association of ulceration of the foot with cardiovascular and all-cause mortality in patients with diabetes: a meta-analysis. Diabetologia 201255 2906-2912. (doi:10.1007/s00125012-2673-3) 